Published in final edited form as:

J Affect Disord. 2020 January 01; 260: 73-76. doi:10.1016/j.jad.2019.09.005.

\title{
A Test of the Interpersonal Theory of Suicide in College Students
}

\author{
Stephen P. Becker ${ }^{1,2}$, Josalyn A. Foster ${ }^{1}$, Aaron M. Luebbe ${ }^{3}$ \\ ${ }^{1}$ Division of Behavioral Medicine and Clinical Psychology, Center for ADHD, Cincinnati Children's \\ Hospital Medical Center, Cincinnati, Ohio, USA \\ 2Department of Pediatrics, University of Cincinnati College of Medicine, Cincinnati, Ohio, USA \\ ${ }^{3}$ Department of Psychology, Miami University, Oxford, Ohio, USA
}

\begin{abstract}
Background: The interpersonal theory of suicide posits that perceived burdensomeness, thwarted belongingness, and acquired capability together interact to increase risk for lethal selfinjury. Despite the prominence of this theoretical model, few studies have directly tested the threeway interaction central to the theory, with mixed findings reported in studies to date. The objective of this study was to test the theorized three-way interaction in relation to suicidal behaviors in a large sample of college students.
\end{abstract}

Methods: Undergraduate students were recruited from two universities $(N=1,686$; ages 18-29; $64.5 \%$ female). Participants completed measures of perceived burdensomeness and thwarted belongingness (Interpersonal Needs Questionnaire-25), fearlessness about death (Acquired Capability for Suicide Scale-Fearlessness About Death), and suicidal behaviors (Suicidal Behaviors Questionnaire-Revised).

Results: Multiple linear regression was used to test the three-way interaction among burdensomeness, belongingness, and fearlessness about death on suicidal behaviors. Controlling for sex and depressive symptoms, results indicated the presence of a significant three-way interaction. The interaction was probed by dichotomizing burdensomeness at high and low values. There was a significant two-way interaction at high burdensomeness such that low belongingness was only related to suicidal behavior at high levels of fearlessness about death.

\footnotetext{
Address correspondence to: Stephen Becker, Center for ADHD, Division of Behavioral Medicine and Clinical Psychology, Cincinnati Children's Hospital Medical Center, 3333 Burnet Avenue, MLC 10006, Cincinnati, Ohio 45229-3039; (513) 803-2066 (phone); stephen.becker@cchmc.org.

Contributors

Stephen P. Becker was the primary author of the manuscript and contributed the majority of the writing and is a PI on the study from which the data was sourced. Josalyn A. Foster contributed to the literature search and manuscript writing and refinement. Aaron M. Luebbe completed manuscript analyses, contributed to manuscript revision and refinement and is a PI on the study from which the data was sourced. All authors have approved the final article and agreed to authorship order.

Publisher's Disclaimer: This is a PDF file of an unedited manuscript that has been accepted for publication. As a service to our customers we are providing this early version of the manuscript. The manuscript will undergo copyediting, typesetting, and review of the resulting proof before it is published in its final citable form. Please note that during the production process errors may be discovered which could affect the content, and all legal disclaimers that apply to the journal pertain.

Declarations of Interest: None.

Conflict of interests: None.
} 
Limitations: The study is cross-sectional and uses a composite measure of suicidal behaviors.

Conclusions: This study adds to a small but growing body of research testing the three-way interaction among perceived burdensomeness, thwarted belongingness, and capability for suicide in relation to suicidal behaviors. Longitudinal studies using measures that distinguish between suicidal ideation and suicide attempt within the ideation-to-action framework are needed.

\section{Keywords}

acquired capability; interpersonal-psychological theory; perceived burdensomeness; suicide; thwarted belongingness; university students

Suicide is a major public health concern worldwide and is the second leading cause of death among young adults, including college students (Centers for Disease Control and Prevention, 2015; World Health Organization, 2014). It is therefore imperative to build and examine models that can inform our understanding of the full continuum of suicidal thoughts and behaviors (O'Connor and Nock, 2014). One prominent model is the interpersonal theory of suicide (Joiner, 2005; Van Orden et al., 2010), which posits that hopelessness about states of thwarted belongingness (e.g., "I feel disconnected from others") and perceived burdensomeness (e.g., "I am a burden on society") together contribute to suicidal desire (suicidal ideation). An acquired capability for suicide, which lowers fear of death and increases physical pain tolerance, is theorized to increase the likelihood that an individual will transition from suicidal desire to suicidal intent including lethal or non-lethal suicide attempts (Joiner, 2005; Van Orden et al., 2010).

There is growing empirical support for the interpersonal theory of suicide (Chu et al., 2017; Ma et al., 2016). However, very few studies have directly tested a central hypothesis of the theory wherein thwarted belongingness, perceived burdensomeness, and capability for suicide will interact to predict suicidal behaviors. A recent systematic review of the theory's predictions found that only a small fraction (5.8\%) tested the hypothesized three-way interaction (Ma et al., 2016). A few studies have found the three-way interaction to predict increased suicidal behaviors as expected (Anestis and Joiner, 2011; Anestis et al., 2015; Davidson et al., 2010; Glaesmer et al., 2017; Joiner et al., 2009), whereas others have not (Bryan et al., 2010; Czyz et al., 2015; Monteith et al., 2013). Potentially, nonsignificant findings were at least partly attributable to sample size limitations (Ma et al., 2016). In addition, it appears that the three-way interaction may not be specific to lethal or non-lethal suicide attempts as originally posited by the theory, but rather predicts a broader range of suicidal behaviors such as ideation (Chu et al., 2017; Ma et al., 2016). The purpose of the present study was to test the three-way interaction of the interpersonal theory of suicide in relation to suicidal behaviors in a large sample of college students, controlling for sex and depressive symptoms since female sex and higher depressive symptoms are related to increased suicidal behaviors (Becker et al., 2018; Wilcox et al., 2010). Given the interpersonal model of suicide's predictions (Joiner, 2005; Van Orden et al., 2010), and recent reviews of the empirical literature (Chu et al., 2017; Ma et al., 2016), we hypothesized that the three-way interaction would significantly predict higher scores on a composite measure of suicidal behaviors. 


\section{Methods}

\section{Participants}

Participants were 1,696 college students enrolled in two public universities in the Midwest United States. Participants ranged in age from 18 to 29 years. There were 1,094 women, 594 men, and 6 participants who indicated their sex as Other. The majority $(81.7 \%, n=1,385)$ of participants self-identified as White; the remaining participants self-identified as Asian/ Asian American (7.7\%, $n=130)$, Black/African American (5.8\%, n=99), Biracial/Multiracial $(4.0 \%, n=68)$, American Indian/Alaska Native $(0.4 \%, n=6)$, or Native Hawaiian/Other Pacific Islander $(0.1 \%, n=2)$. Seventy-three participants (4.3\%) self-identified as Hispanic/ Latino. The sex, race, and ethnicity items were not completed by 2, 6, and 2 participants, respectively. Most participants $(63.4 \%)$ were in their first year of college; the remaining participants were in their second (23.6\%), third (8.7\%), fourth $(4.0 \%)$, or other $(0.2 \%)$ year of college.

\section{Procedures}

This study was approved by the Institutional Review Board (IRB) at each institution. Students enrolled in introductory psychology courses were able to participate in a research study to fulfill a course requirement. Students were able to choose from a number of studies and could choose to participate if this study if they were $\geq 18$ years old. Participants at each institution completed the survey in Qualtrics anonymously and received course credit for participation. All participants received the contact information of the local investigator, IRB, and student counseling center. For additional details, see (Becker et al., 2018).

\section{Measures}

Interpersonal Needs Questionnaire.-The Interpersonal Needs Questionnaire (INQ) (Van Orden et al., 2012) is a 25-item self-report measure that includes 10 items assessing thwarted belongingness and 15 items assessing perceived burdensomeness (Van Orden et al., 2012). Participants indicate how true each item is for them on a seven-point scale, with higher scores indicating greater thwarted belongingness and perceived burdensomeness. In the present study, Cronbach's as $=.89$ and .96 for thwarted belongingness and perceived burdensomeness, respectively.

\section{Acquired Capability for Suicide Scale-Fearlessness About Death.-The} Acquired Capability for Suicide Scale-Fearlessness About Death (ACSS-FAD) is derived from the Acquired Capability for Suicide Scale (Ribeiro et al., 2014). The ACSS-FAD consists of seven items rated on a four-point scale, with higher scores representing more fearlessness about death (Ribeiro et al., 2014). In the present study, Cronbach's a $=.80$.

Suicidal Behaviors Questionnaire-Revised.-The Suicidal Behaviors QuestionnaireRevised (SBQ-R) (Osman et al., 2001) is a validated and reliable measure of suicidal behaviors (Osman et al., 2001). The SBQ-R includes four items that assess lifetime ideation/ attempt, frequency of ideation over the past 12 months, telling someone else about ideation, and likelihood of attempting suicide in the future. The SBQ-R is the most frequently-used 
composite measure of suicidal behaviors/suicide risk (Chu et al., 2017; Ma et al., 2016). In the present study, Cronbach's $\alpha=.82$.

Depression Anxiety Stress Scales.-The depression subscale of the Depression Anxiety Stress Scales-21 (DASS-21; (Antony et al., 1998; Lovibond and Lovibond, 1995) was used to assess depressive symptoms. The depression subscale consists of seven items, none of which focus on suicide or sleep. Each item is rated using a four-point scale $(0=$ did not apply to me at all, $3=$ applied to me very much or most of the time). In the present study, Cronbach's a $=.90$.

\section{Results}

\section{Missing Data}

A total of 1,704 participants completed the study. Overall, there was very little missing data $\left(0.29 \%\right.$ of all data; Little's MCAR $\chi^{2}(6)=5.09, p=.532$, suggesting data were missing in a pattern consistent with missing completely at random). Eight participants were missing data on all three interpersonal theory of suicide variables. Given our focus on these three scores and the small loss of data, we chose to listwise delete these individuals. Once removing these data, we used the EM algorithm to impute a single value for the remaining missing values (i.e., two values for INQ-Burdensomeness and one value for INQ-Belongingness). The sample description above and the analyses below are based on the final sample of 1,696 participants.

\section{Preliminary Analyses}

Descriptive data and intercorrelations of all study measures are in Table 1 . With the exception of age (skew $=2.45$; kurtosis $=10.21$ ), all data were within acceptable limits of skew $(<2.0)$ and kurtosis $(<4.0)$. Two small effects between demographic data and our primary outcome, suicide risk, were noted. First, suicide risk scores were significantly higher at one of our two data collection sites than the other. Second, women endorsed significantly higher suicide risk than men. This effect was small. Given these two significant effects, site and sex were included as covariates in primary analyses. Because there were only eight participants who identified as "other" when asked about sex, we did not include these individuals in our preliminary bivariate analyses. So as not to exclude these individuals from our sample, however, sex was accounted for by two dummy-coded vectors in primary analyses, with men as the reference group. Racial and ethnic minority / majority status and age were not related to suicidal behaviors and were not considered further. There were moderate to strong intercorrelations among low belongingness, high burdensomeness, depressive symptoms, and suicide risk. Although significant, the relation of fearlessness about death to suicide risk was quite small. Fearlessness about death was unrelated to belongingness, burdensomeness, and depression.

\section{Primary Analyses}

Multiple linear regression was used to test the three-way interaction among burdensomeness, low belongingness, and fearlessness about death on suicidal behavior. Controlling for data collection site, participant sex, and depression, results indictated the presence of a significant 
three-way interaction (see Table 2). Because of a floor effect on burdensomeness, we probed the interaction by dichotimizing burdensomeness at high and low values (i.e., $-1 \mathrm{SD}$ and mean values on burdensomessness were identical given the distribution of this variable; see Figure 1). Examining two way interactions at these two levels, there was a significant twoway interaction at high burdensomeness $(b=.23, p=.010)$, but not at low levels ( $b=.04, p$ $=.526$ ). Simple slopes analyses, probing the interaction at $+1 \mathrm{SD}$, mean, and $-1 \mathrm{SD}$ values on fearlessness about death indicated a disordinal interaction. Consistent with hypotheses, low belonginess was only positively related to suicidal behavior at high levels of burdensomeness and high levels of fearlessness about death (at +1SD, $b=.20, p=.065$ ).

Given the disordinal interaction, it would appear that at high levels of burdensomeness but when fearlessness is low, that low belongingess might actually be protective (i.e., an inverse relation). As such, it appears in Figure 1 that one would expect some of the highest rates of suicidal behavior from particiants with low belongingness, low fearlessness, but high burdensomeness. However, only 1 participant in our sample of 1,696 would qualify for this data point.

\section{Discussion}

This study adds to a small but growing body of research directly testing the hypothesized three-way interaction among perceived burdensomeness, thwarted belongingness, and capability for suicide that is central to the interpersonal theory of suicide. Most previous studies finding support for this three-way interaction have used higher-risk clinical/military samples (Anestis and Joiner, 2011; Anestis et al., 2015; Joiner et al., 2009). The present study is, to our knowledge, only the second study to examine and find support for the threeway interaction in college students specifically, with the previous college student study comprised of 115 Black students attending a leadership conference (Davidson et al., 2010). Thus, our study provides an important replication in a larger, predominantly White sample from two universities. These findings provide important support for aspects of the interpersonal theory of suicide, though we note that we did not examine the key aspect of the theory that is specific to lethal or near-lethal suicide attempts. The SBQ-R used in this study is a composite measure that includes various features of suicidality, and studies are needed that include measures and methods that can distinguish between suicidal ideation and suicide attempt within the ideation-to-action framework (Klonsky et al., 2016). It will be important for future studies to also include other key constructs such as withdrawal (Joiner et al., 2016; Van Orden et al., 2010) and emotion dysregulation (Law et al., 2015).

Limitations of our study include the cross-sectional design that precludes drawing causal or temporal conclusions and a limited measurement of suicidal behaviors. Although there is evidence that the three-way interaction may not be specific to lethal or near-lethal suicide attempts as posited by the interpersonal theory of suicide (Chu et al., 2017; Ma et al., 2016), this is an especially important area for investigation, including among college students. Prospective longitudinal research is also sorely needed, as very few studies have tested the three-way interaction in studies using a longitudinal design (Czyz et al., 2015). Nevertheless, findings from the present study using a large sample to test a theory-driven hypothesis 
provide important support for the combination of thwarted belongingness, perceived burdensomeness, and fearlessness about death in relation to increased suicidal behaviors.

\section{Acknowledgements:}

Stephen Becker is currently supported by award K23MH108603 from the National Institute of Mental Health (NIMH). The funding source had no involvement in the study design; collection, analysis, and interpretation of data; writing of the manuscript; or the decision to submit the article for publication. The content is solely the responsibility of the authors and does not necessarily represent the official views of the National Institutes of Health (NIH).

Role of the Funding Source

Stephen Becker is currently supported by award number K23MH108603 from the National Institute of Mental Health (NIMH). The content is solely the responsibility of the authors and the National Institutes of Health (NIH) did not contribute to the research or manuscript.

\section{References}

1. Anestis MD, Joiner TE, 2011 Examining the role of emotion in suicidality: negative urgency as an amplifier of the relationship between components of the interpersonal-psychological theory of suicidal behavior and lifetime number of suicide attempts. Journal of affective disorders 129, 261269. [PubMed: 20825997]

2. Anestis MD, Khazem LR, Mohn RS, Green BA, 2015 Testing the main hypotheses of the interpersonal-psychological theory of suicidal behavior in a large diverse sample of United States military personnel. Comprehensive psychiatry 60, 78-85. [PubMed: 25873432]

3. Antony MM, Bieling PJ, Cox BJ, Enns MW, Swinson RP, 1998 Psychometric properties of the 42item and 21-item versions of the Depression Anxiety Stress Scales in clinical groups and a community sample. Psychological assessment 10, 176-181.

4. Becker SP, Holdaway AS, Luebbe AM, 2018 Suicidal behaviors in college students: Frequency, sex differences, and mental health correlates including sluggish cognitive tempo. The Journal of adolescent health : official publication of the Society for Adolescent Medicine 63, 181-188. [PubMed: 30153929]

5. Bryan CJ, Morrow CE, Anestis MD, Joiner TE, 2010 A preliminary test of the interpersonalpsychological theory of suicidal behavior in a military sample. Pers Indiv Differ 48, 347-350.

6. Centers for Disease Control and Prevention, 2015 Web-baased injury statisics query and reporting system (WISQARS), Leading casues of death reports, 1981-2015. Center for Disease Control and Prevention.

7. Chu C, Buchman-Schmitt JM, Stanley IH, Hom MA, Tucker RP, Hagan CR, Rogers ML, Podlogar MC, Chiurliza B, Ringer FB, Michaels MS, Patros CHG, Joiner TE, 2017 The interpersonal theory of suicide: A systematic review and meta-analysis of a decade of cross-national research. Psychological bulletin 143, 1313-1345. [PubMed: 29072480]

8. Czyz EK, Berona J, King CA, 2015 A prospective examination of the interpersonal-psychological theory of suicidal behavior among psychiatric adolescent inpatients. Suicide \& life-threatening behavior 45, 243-259. [PubMed: 25263410]

9. Davidson CL, Wingate LR, Slish ML, Rasmussen KA, 2010 The Great Black Hope: Hope and its relation to suicide risk among African Americans. Suicide \& life-threatening behavior 40, 170-180. [PubMed: 20465352]

10. Glaesmer H, Hallensleben N, Forkmann T, Spangenberg L, Kapusta N, Teismann T, 2017 Testing the main prediction of the Interpersonal Theory of Suicide in a representative sample of the German general population. Journal of affective disorders 211, 150-152. [PubMed: 28126614]

11. Joiner T, 2005 Why people die by suicide. Harvard University Press, Cambridge, MA.

12. Joiner TE, Hom MA, Hagan CR, Silva C, 2016 Suicide as a derangement of the self-sacrificial aspect of eusociality. Psychological review 123, 235-254. [PubMed: 26524155] 
13. Joiner TE, Van Orden KA, Witte TK, Selby EA, Ribeiro JD, Lewis R, Rudd MD, 2009 Main predictions of the interpersonal-psychological theory of suicidal behavior: empirical tests in two samples of young adults. Journal of abnormal psychology 118, 634-646. [PubMed: 19685959]

14. Klonsky ED, May AM, Saffer BY, 2016 Suicide, suicide attempts, and suicidal ideation. Annual review of clinical psychology 12, 307-330.

15. Law KC, Khazem LR, Anestis MD, 2015 The role of emotion dysregulation in suicide as considered through the ideation to action framework. Current opinion in psychology 3, 30-35.

16. Lovibond PF, Lovibond SH, 1995 The structure of negative emotional states: Comparison of the Depression Anxiety Stress Scales (DASS) with the Beck Depression and Anxiety Inventories. Behaviour research and therapy 33, 335-343. [PubMed: 7726811]

17. Ma J, Batterham PJ, Calear AL, Han J, 2016 A systematic review of the predictions of the Interpersonal-Psychological Theory of Suicidal Behavior. Clinical psychology review 46, 34-45. [PubMed: 27155061]

18. Monteith LL, Menefee DS, Pettit JW, Leopoulos WL, Vincent JP, 2013 Examining the interpersonal-psychological theory of suicide in an inpatient veteran sample. Suicide \& lifethreatening behavior 43, 418-428. [PubMed: 23556542]

19. O'Connor RC, Nock MK, 2014 The psychology of suicidal behaviour. The lancet. Psychiatry 1, 73-85. [PubMed: 26360404]

20. Osman A, Bagge CL, Gutierrez PM, Konick LC, Kopper BA, Barrios FX, 2001 The Suicidal Behaviors Questionnaire-Revised (SBQ-R): validation with clinical and nonclinical samples. Assessment 8, 443-454. [PubMed: 11785588]

21. Ribeiro JD, Witte TK, Van Orden KA, Selby EA, Gordon KH, Bender TW, Joiner TE Jr., 2014 Fearlessness about death: the psychometric properties and construct validity of the revision to the acquired capability for suicide scale. Psychological assessment 26, 115-126. [PubMed: 24274043]

22. Van Orden KA, Cukrowicz KC, Witte TK, Joiner TE, 2012 Thwarted belongingness and perceived burdensomeness: construct validity and psychometric properties of the Interpersonal Needs Questionnaire. Psychological assessment 24, 197-215. [PubMed: 21928908]

23. Van Orden KA, Witte TK, Cukrowicz KC, Braithwaite SR, Selby EA, Joiner TE Jr., 2010 The interpersonal theory of suicide. Psychological review 117, 575-600. [PubMed: 20438238]

24. Wilcox HC, Arria AM, Caldeira KM, Vincent KB, Pinchevsky GM, O’Grady KE, 2010 Prevalence and predictors of persistent suicide ideation, plans, and attempts during college. Journal of affective disorders 127, 287-294. [PubMed: 20471691]

25. World Health Organization, 2014 Preventing suicide: A global imperative. World Health Organization, Geneva, Switzerland. 


\section{Highlights}

- The interpersonal theory of suicide needs additional tests of key hypotheses.

- A three-way interaction provided support for the interpersonal theory of suicide.

- Low belongingness related to suicidal behavior at high fearlessness about death.

- Longitudinal studies and nuanced measurement are needed to build upon findings. 


\section{Test of the Interpersonal Model of Suicide}

7.00

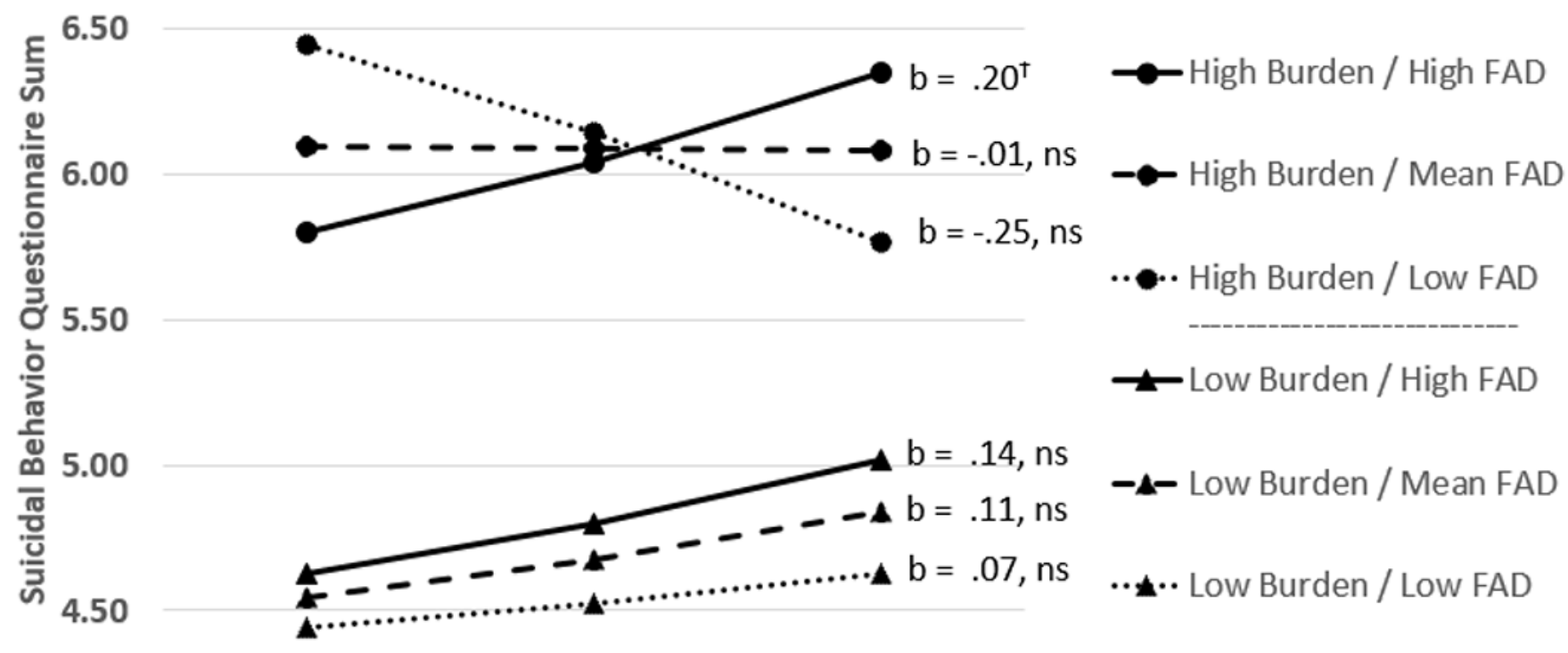

4.00

$-1 S D$

MEAN

+1SD

Low Belongingness

Figure 1.

Test of the three-way interaction among belongingness, burdensomeness, and fearlessness about death in relation to suicidal behavior. ${ }^{\dagger} p<.07$. 


\section{Table 1}

Intercorrelations, Means, Standard Deviations, and Range of Study Variables

\begin{tabular}{|c|c|c|c|c|c|c|c|c|c|}
\hline & Site & Age & Sex & Race & INQ-Burden. & INQ-Belong. & FAD & $\begin{array}{c}\text { DASS } \\
\text { DEP }\end{array}$ & SBQ-R \\
\hline Age & $.11^{* * *}$ & - - & & & & & & & \\
\hline Sex & .01 & $-.17^{* *}$ & - & & & & & & \\
\hline Race & -.01 & $-.07^{* * *}$ & .01 & -- & & & & & \\
\hline INQ-Burden. & $.07^{* *}$ & -.01 & -.02 & $-.08^{* *}$ & -- & & & & \\
\hline INQ-Belong. & $.08^{* * *}$ & $.05^{*}$ & $-.08^{* * *}$ & $-.12^{* * *}$ & $.59^{* * *}$ & - & & & \\
\hline FAD & -.02 & $.07^{* *}$ & $-.17^{* *}$ & -.04 & .03 & .04 & - & & \\
\hline DASS DEP & $.06^{*}$ & .01 & -.01 & $-.06^{*}$ & $.55^{* *}$ & $.54^{* *}$ & .03 & - & \\
\hline SBQ-R & $.12^{* *}$ & -.01 & $.05^{*}$ & -.04 & $.52^{* *}$ & $.41^{* *}$ & $.06^{* *}$ & $.55^{* *}$ & - \\
\hline$M$ & 0.50 & 18.98 & 0.65 & 0.82 & 1.69 & 2.70 & 1.98 & 0.57 & 5.17 \\
\hline$S D$ & -- & 1.27 & -- & -- & 1.14 & 1.24 & 0.92 & 0.62 & 2.82 \\
\hline Range & $0-1$ & $18-29$ & $0-1$ & $0-1$ & $1.00-7.00$ & $1.00-6.67$ & $0.00-4.00$ & $0.00-3.00$ & $3.00-18.00$ \\
\hline
\end{tabular}

Note. For most correlations $\mathrm{N}=1,696$. For those with sex, $\mathrm{N}=1,688$, and race, $\mathrm{N}=1690$. For sex, male $=0$, female $=1$. For race, racial $/$ ethnic minority $=0$, White $=1$. INQ-Burden $=$ Interpersonal Needs Questionnaire - Burdensomeness subscale. INQ-Belong $=$ Interpersonal Needs Questionnaire - Belongingness subscale. FAD = Fearless About Death scale. DASS DEP = Depression, Anxiety, Stress Scale - Depression subscale. SBQ = Suicide Behaviors Questionnaire-Revised.

* $p<.05$.

** $p<.01$. 


\section{Table 2}

Testing the Interaction of Low Belongingness, Burdensomeness, and Fearlessness about Death in relation to Suicide Risk $(\mathrm{N}=1,696)$

\begin{tabular}{|c|c|c|c|c|c|}
\hline DV = SBQ-R & $b$ & $S E$ & $\beta$ & $t$ & $s r$ \\
\hline \multicolumn{6}{|c|}{$F(11,1684)=94.90, p<.001 . R^{2}=.38$} \\
\hline Site & 0.43 & .11 & .08 & $4.02^{* *}$ & .08 \\
\hline Sex_D1 & 1.41 & .79 & .03 & 1.78 & .03 \\
\hline Sex_D2 & 0.41 & .12 & .07 & $3.54^{* *}$ & .07 \\
\hline Depression & 1.66 & .11 & .36 & $14.90^{* *}$ & .29 \\
\hline Low Belongingness & 0.06 & .06 & .03 & 1.07 & .02 \\
\hline Burdensomeness & 0.76 & .08 & .31 & $9.23^{* *}$ & .18 \\
\hline Fearlessness About Death & 0.09 & .07 & .03 & 1.23 & .02 \\
\hline Belong x Burden & -0.06 & .05 & -.04 & -1.27 & -.02 \\
\hline Belong x FAD & 0.11 & .06 & .05 & 1.92 & .04 \\
\hline Burden $x$ FAD & -0.10 & .09 & -.03 & -1.10 & -.02 \\
\hline Belong $\mathrm{x}$ Burden $\mathrm{x} F A D$ & 0.11 & .05 & .07 & $2.27^{*}$ & .04 \\
\hline
\end{tabular}

INQ-Burden = Interpersonal Needs Questionnaire - Burdensomeness subscale. INQ-Belong = Interpersonal Needs Questionnaire - Belongingness subscale. FAD $=$ Fearless About Death scale. DASS DEP $=$ Depression, Anxiety, Stress Scale - Depression subscale. SBQ-R $=$ Suicide Behaviors Questionnaire-Revised.

*

$p<.05$.

*** $p<.01$ 